\title{
Chest Wall Silicone Granuloma Following Ruptured Silicone Breast Implant Causes Giant Chest Wall Abscess and Osteomyelitis: The First Report
}

\author{
(1) Hanad Ahmed ${ }^{1,2}$, (1) Alessandro Tamburrini' ${ }^{2}$, (1) Mansoor Khan ${ }^{3}$, (1) Aiman Alzetani² \\ ${ }^{1}$ University of Southampton, Faculty of Medicine, Southampton, United Kingdom \\ ${ }^{2}$ University Hospital Southampton, Thoracic unit, Southampton, United Kingdom \\ ${ }^{3}$ University Hospital Southampton, Plastics and Reconstructive Unit, Southampton, United Kingdom
}

\begin{abstract}
Silicone breast implant ruptures have been widely reported in the literature. Granuloma formation is a known complication of such ruptures with reported sites including the axillae, limbs, chest wall muscles, and internal organs, such as the lungs and the liver. To the best of our knowledge, there are no reported cases of a silicone granuloma causing osteomyelitis of the sternum and multiple ribs in the absence of infection. We therefore report on the case of an 81-year-old patient who presented with an anterior chest wall discharging sinus tract on the background of a ruptured silicone breast implant. We raise awareness about the potentially devastating complications resulting from a ruptured silicone implant with relevance to cardiothoracic practice.
\end{abstract}

Keywords: Silicone breast implant, rupture, granuloma, chest wall, cardiothoracic

Cite this article as: Ahmed H, Tamburrini A, Khan M, Alzetani A. Chest Wall Silicone Granuloma Following Ruptured Silicone Breast Implant Causes Giant Chest Wall Abscess and Osteomyelitis: The First Report. Eur J Breast Health 2021; 17(4): 383-385.

\section{Key Points}

- Recognition of this rare but serious complication.

- Early intervention is vital to avoid significant chest wall destruction.

- Ensure meticulous surgical debridement of all involved tissue to a healthy, bleeding margin.

- $\quad$ Reconstruct with a well-vascularized, preferably pedicled, graft to protect against any infection of the composite prosthesis.

\section{Introduction}

Silicone breast implants have been used extensively in cosmetic and reconstructive surgery for over 50 years (1). Implant ruptures are a known complication and likely have an underestimated incidence due to their asymptomatic nature (2). In most cases, the leak of silicone gel remains confined within the intact fibrous capsule. However, in $12 \%$ to $26 \%$ of cases, silicone may spread beyond the capsule and reach the breast parenchyma, termed an extracapsular rupture (3), potentially causing foreign body inflammation leading to a silicone granuloma (4). Rare cases of silicone granuloma involving the axillae, limbs, chest wall muscles, liver, lung, abdominal wall, and inguinal area have been described (5-11). However, to the best of our knowledge, this is the first report of an advanced silicone granuloma causing osteomyelitis of the ribs and sternum.

\section{Case Presentation}

An 81-year-old female with a history of bilateral breast cancer was referred to our tertiary centre with a recalcitrant, right-sided, chest wall granuloma and chronic sternal osteomyelitis unresponsive to antibiotic therapy. This visit followed the removal of a ruptured right silicone implant. On inspection, the patient had extensive chest wall scarring from previous surgeries and radiation injuries to her neck and precordial area. She had skin loss around the right sternal edge, medial to her breast scar, with underlying bone exposure and a discharging sinus area, as illustrated in Figure 1. On examination, she was tender over the right sternal edge but was otherwise asymptomatic. Her observations were unremarkable, and laboratory investigations were significant for C-reactive protein of $182 \mathrm{mg} / \mathrm{L}$. Swabs from the wound site revealed no growth of microorganisms. Her biopsy showed no signs of cancer but concurred with imaging studies showing osteomyelitis of the surrounding bone. 


\section{Surgical management}

The patient's surgical management involved both the cardiothoracic and plastics and reconstructive teams. She was admitted for an elective anterior chest wall resection involving the sternal body and ribs 2-6, including the overlying skin and subcutaneous fat, as illustrated in Figure 2. Intra-operative findings included abnormal soft tissue extending down to the ribs with adhesions to the lung and mediastinal fat, illustrated in Figure 3. The reconstruction was performed using a Marlex mesh sandwich reinforced with gentamicin cement to repair the bony defect. This was covered with a pedicled latissimus dorsi myocutaneous flap to replace the soft tissue loss. The result is shown in Figure 4.

Histopathological evaluation revealed tissue with abundant inflammatory cells, hemosiderin-laden macrophages, and foreignbody giant cells. Sections from decalcified ribs showed areas of necrosis surrounding fibrocollagenous tissue and dead bony trabeculae, confirming the chronic sinus tract clinical diagnosis. There was no evidence of malignancy.

The operation and postoperative course were uneventful, and the patient was discharged home with long-term antibiotics. She was seen in the clinic three months after inpatient discharge, showing good wound healing without evidence of cellulitis or local tenderness.

\section{Discussion and Conclusion}

The design and materials science of silicone implants have evolved considerably over the past four decades (12). The implant's shape and filler, and shell characteristics have changed drastically to minimize implant compromise (12). Despite improvements in implant stability, ruptured silicone breast implant cases are still reported in the literature. Rupture incidences have been shown to increase with implant age, particularly after the 6-year mark (13). The cumulative

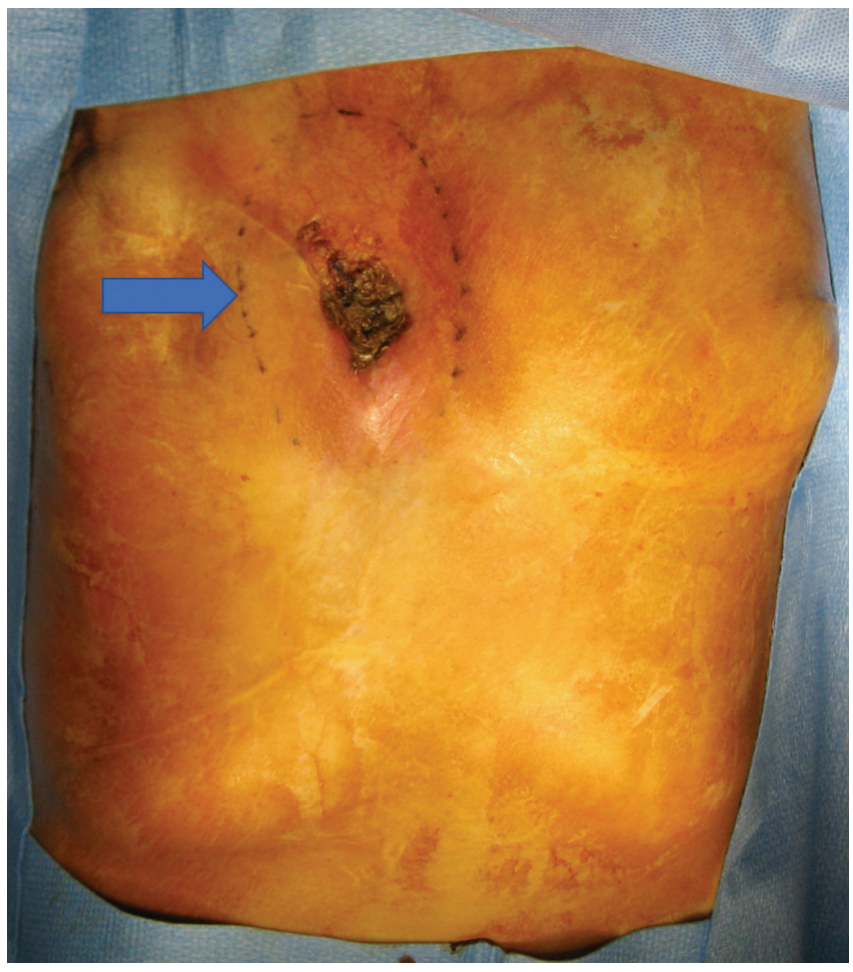

Figure 1. Image illustrating the discharging sinus area over the right sternal margin overall rupture incidence at six years for patients undergoing primary reconstruction after a mastectomy is $1.5 \%$ or $3.8 \%$, although this figure varies depending on the implant manufacturer (1). Some proposed rupture mechanisms include trauma to the chest, damage caused by surgical instruments, and implant shell swelling (1). Upon rupture, the patient's body creates a fibrous capsule around the foreign silicone material (1). A rupture still contained within this capsule is termed an intracapsular rupture (1). A breach of this capsule results in an extracapsular rupture and allows the silicone to migrate to distant tissues (1). If the silicone is not removed, a chronic inflammatory state ensues, often resulting in local tissue destruction, as seen in our patient.

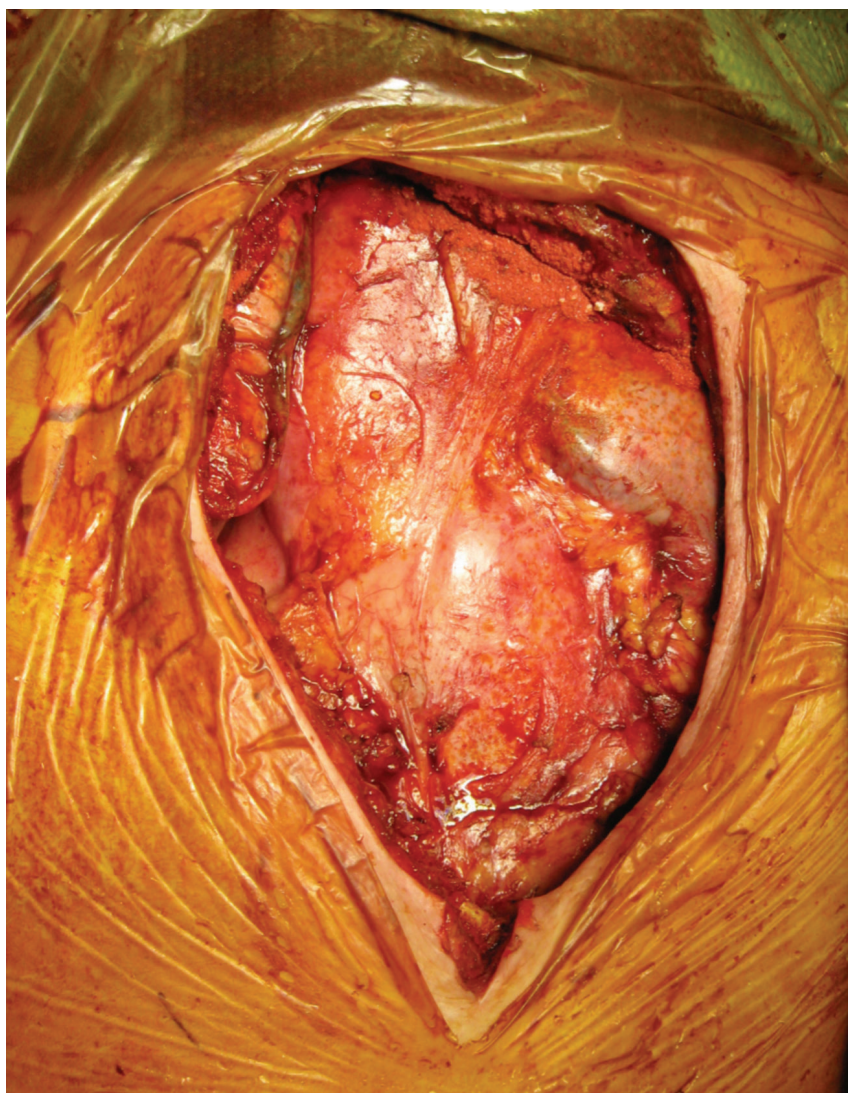

Figure 2. Site of anterior chest wall resection

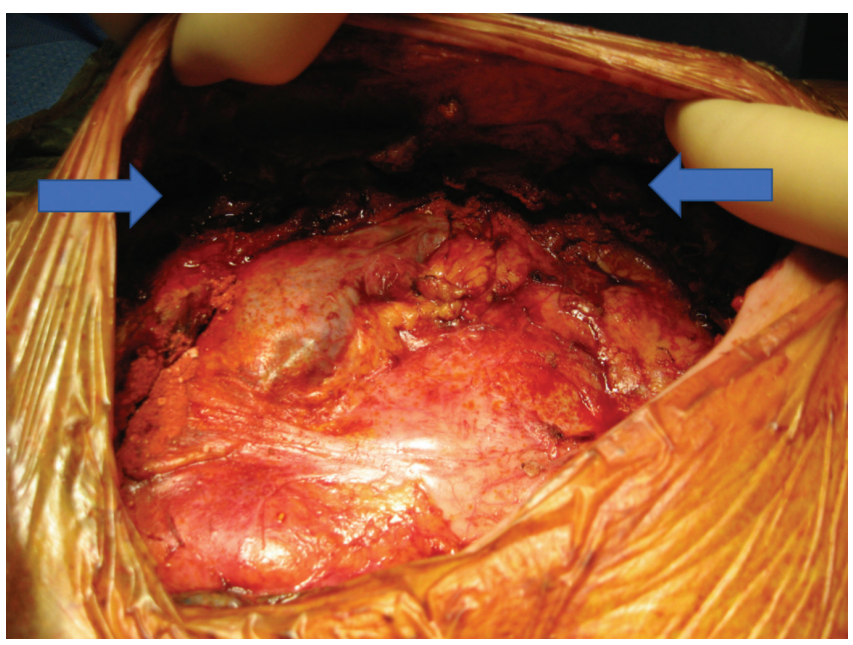

Figure 3. Extension of abnormal soft tissue into the chest wall 


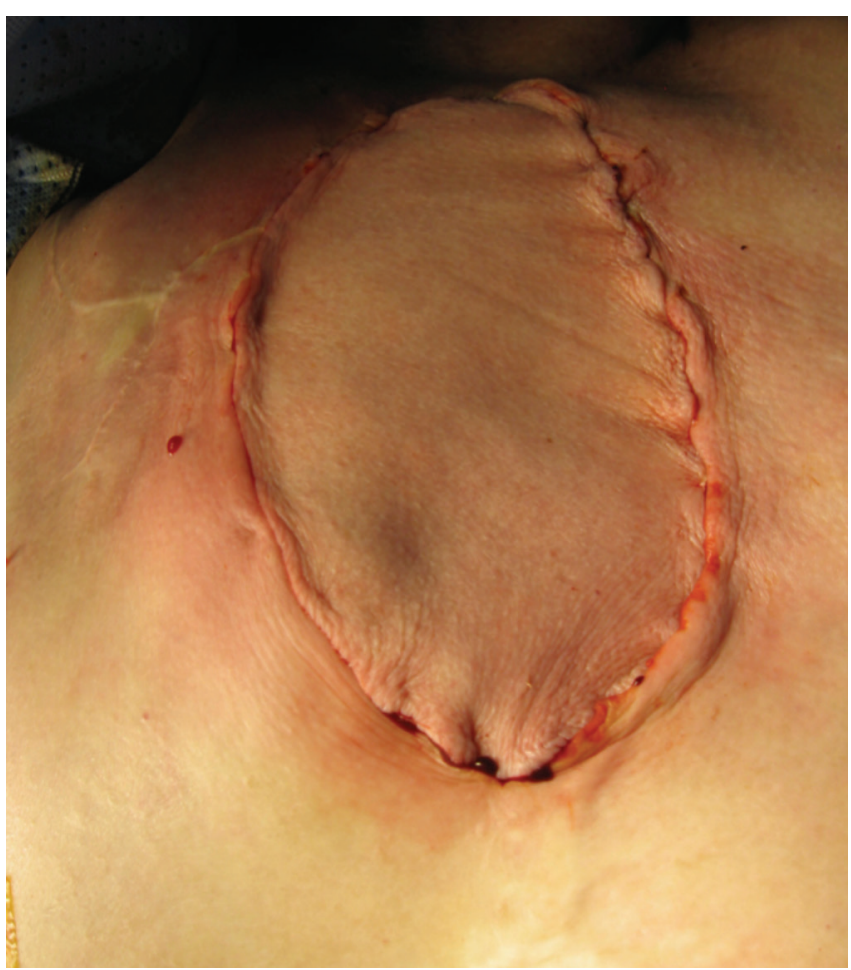

Figure 4. Post-reconstruction with latissimus dorsi myocutaneous flap

Although extremely rare, osteomyelitis of the ribs and sternum, secondary to a breast implant, has previously been reported in the context of implant infection directly spreading to bone (14). However, in our patient, wound cultures were negative for microorganisms, limiting the tissue destruction's aetiology to granulomatous inflammation due to the silicone particles inflammation.

In conclusion, although our patient recovered well, this case highlights the potential complications resulting from extracapsular silicone implant ruptures and their relevance to cardiothoracic surgery. Early diagnosis and management are vital in preventing granuloma formation and potential tissue destruction. The low incidence of these complications, patients' asymptomatic nature, and the low sensitivity of physical examinations make it challenging to detect silent ruptures. Therefore, clinicians should be aware of the potential complications of ruptured silicone implants and exercise a low threshold for imaging studies to ensure early intervention as appropriate for each patient.

Informed Consent: Consent for research and pictures were obtained from the patient.

Peer-review: Externally peer-reviewed.

\section{Author Contributions}

Conception: H.A., A.T., M.K., A.A.; Design: H.A., A.T., M.K., A.A.; Supervision: A.T., A.A.; Data Collection and/or Processing: H.A.; Literature Search: H.A.; Writing: H.A.; Critical Review: H.A., A.T., M.K., A.A.
Conflict of Interest: The authors have no conflicts of interest to declare.

Financial Disclosure: The authors declared that this study has received no financial support.

\section{References}

1. Hillard C, Fowler JD, Barta R, Cunningham B. Silicone breast implant rupture: a review. Gland Surg 2017: 6: 163-168. (PMID: 28497020) [Crossref]

2. Brown SL, Middleton MS, Berg WA, Soo MS, Pennello G. Prevalence of rupture of silicone gel breast implants revealed on MR imaging in a population of women in Birmingham, Alabama. AJR Am J Roentgenol 2000; 175: 1057-1064 (PMID: 11000165) [Crossref]

3. Berg WA, Nguyen TK, Middleton MS, Soo MS, Pennello G, Brown SL. MR imaging of extracapsular silicone from breast implants: diagnostic pitfalls. AJR Am J Roentgenol 2002; 178: 465-472. (PMID: 11804919) [Crossref]

4. Winer LH, Sternberg TH, Lehman R, Ashley FL. Tissue reactions to injected silicone liquids. Arch Dermatol 1964; 90: 588-592. (PMID: 14206865) [Crossref]

5. Delage C, Shane JJ, Johnson FB. Mammary silicone granuloma. Migration of silicone fluid to abdominal wall and inguinal region. Arch Dermatol 1973; 108: 105-107. (PMID: 4268578) [Crossref]

6. Argenta LC. Migration of silicone gel into breast parenchyma following mammary prosthesis rupture. Aesthetic Plast Surg 1983; 7: 253-254. (PMID: 6364741) [Crossref]

7. Sagi L, Baum S, Lyakhovitsky A, Barzilai A, Shpiro D, Trau H, et al. Silicone breast implant rupture presenting as bilateral leg nodules. Clin Exp Dermatol 2009; 34: e99-e101. (PMID: 19438562) [Crossref]

8. Adams ST, Cox J, Rao GS. Axillary silicone lymphadenopathy presenting with a lump and altered sensation in the breast: a case report. J Med Case Rep 2009; 3: 6442. (PMID: 19830102) [Crossref]

9. Dragu A, Theegarten D, Bach AD, Polykandriotis E, Arkudas A, Kneser $\mathrm{U}$, et al, Ingianni G. Intrapulmonary and cutaneous siliconomas after silent silicone breast implant failure. Breast J 2009; 15: 496-499. (PMID: 19624416) [Crossref]

10. Oh JH, Song SY, Lew DH, Lee DW. Distant migration of multiple siliconomas in lower extremities following breast implant rupture: case report. Plast Reconstr Surg Glob Open 2016; 4: e101. (PMID: 27826457) [Crossref]

11. Ikizceli T, Gulsen G, Akin I. Silicone granuloma associated with pectoral puscle involvement after ruptured breast implant: a novel case report. Eur J Breast Health 2018; 14: 54-57 (PMID: 29322121) [Crossref]

12. Maxwell, G.P. and Gabriel, M. Breast implant design. Gland Surg 2017; 6: 148-153. (PMID 28497018) [Crossref]

13. Hölmich LR, Friis S, Fryzek JP, Vejborg IM, Conrad C, Sletting S, et al. Incidence of silicone breast implant rupture. Arch Surg 2003; 138: 801806. (PMID 12860765) [Crossref]

14. Seng P, Alliez A, Honnorat E, Menard A, Casanova D, Stein A. Osteomyelitis of sternum and rib after breast prosthesis implantation: a rare or underestimated infection? IDCases 2015; 2: 31-33. (PMID 26793446) [Crossref] 